\title{
Post-tuberculous lung disease: should we be using Theophylline?
}

\author{
Sumanth Karamchand $^{1} \wedge$, Morne Williams ${ }^{1}$, Poobalan Naidoo $^{2}$, Eric Decloedt $^{3}$, Brian Allwood $^{1}$ \\ ${ }^{1}$ Division of Pulmonology, Western Cape Department of Health, Tygerberg Hospital, Bellville, South Africa; ${ }^{2}$ Department of Informatics, School of \\ Health Professions, Rutgers University, New Jersey, USA; ${ }^{3}$ Division of Clinical Pharmacology, Department of Medicine, Faculty of Medicine and \\ Health Sciences, Stellenbosch University and Tygerberg Hospital, Bellville, South Africa \\ Contributions: (I) Conception and design: B Allwood, S Karamchand, M Williams, E DeCloedt; (II) Administrative support: B Allwood; (III) Provision \\ of study materials or patients: B Allwood; (IV) Collection and assembly of data: S Karamchand, M Williams, B Allwood; (V) Data analysis and \\ interpretation: S Karamchand, P Naidoo, B Allwood; (VI) Manuscript writing: All authors; (VII) Final approval of manuscript: All authors. \\ Correspondence to: Sumanth Karamchand. Stellenbosch University, Tygerberg Hospital, Bellville, South Africa. Email: sum801@gmail.com.
}

\begin{abstract}
Tuberculosis affects 10 million people and over 320,000 South Africans every year. A significant proportion of patients treated for tuberculosis develop post-tuberculous lung disease (PTBLD), a disease of chronic respiratory impairment for which there is a lack of affordable treatment options. PTBLD a heterogenous disorder that shares phenotypical features with chronic obstructive lung disease, bronchiectasis, lung fibrosis and destruction as well as pulmonary hypertension. There remains a paucity of proven pharmacotherapy for the management of PTBLD. Theophylline, a widely available and affordable medicine that has largely fell out of favour in high-income settings due to its toxicity and narrow therapeutic index, may be repositioned for the treatment of PTBLD. In this review, we unpack the potential role of theophylline in the management of PTBLD by reviewing the evidence for its bronchodilatory, antiinflammatory and potential pleotrophic effects.
\end{abstract}

Keywords: Post tuberculous lung disease (PTBLD); theophylline; tuberculosis (TB); chronic obstructive pulmonary disease (COPD); pulmonary hypertension

Submitted Mar 19, 2020. Accepted for publication Oct 15, 2020.

doi: $10.21037 /$ jtd-20-1298

View this article at: http://dx.doi.org/10.21037/jtd-20-1298

\section{Introduction}

Tuberculosis affects 10 million people and over 320,000 South African every year. A significant proportion of these patients will develop post-tuberculous lung disease (PTBLD) secondary to a complex interplay between Mycobacterium tuberculosis, host genetic factors and the immune response which may result in activation of proteases with ensuing lung damage (1).

PTBLD is a blanket diagnosis loosely applied to patients with residual parenchymal destruction and symptoms after tuberculosis, and there is renewed interest in defining the various clinical presentations. PTBLD represents a spectrum of diseases with varying phenotypes that in any individual may share features of chronic obstructive pulmonary disease (COPD), bronchiectasis, lung fibrosis and destruction as well as pulmonary hypertension, with potential ongoing inflammation and associated tissue damage.

There remains a lack of evidence on effective pharmacotherapy for the management of PTBLD. Theophylline may have utility in the treatment of PTBLD given its bronchodilatory, anti-inflammatory and potential pleotrophic effects. Theophylline is frequently used in South African and low to middle income countries (LMIC) for COPD, asthma due to its availability and low cost (2). However, theophylline has largely been abandoned in high-

$\wedge$ ORCID: 0000-0003-4435-6685. 
income countries because of concerns around safety due to its narrow therapeutic index.

Given the lack of alternative, affordable treatment options available for PTBLD in high burden countries, including our own, we review the mechanism of action, and evidence both for and against the use of theophylline in the treatment of COPD, bronchiectasis and pulmonary hypertension, the analogous clinical syndromes of PTBLD. In this review, we discuss the potential role of theophylline in the management of PTBLD, by reviewing the evidence for its bronchodilatory, anti-inflammatory and potential pleotrophic effects.

\section{History of theophylline \& use in SA}

\section{Historical uses}

Theophylline, a dimethylxanthine has been in clinical use for more than 85 years (3). Theophylline occurs naturally in tea and cocoa beans, and was first extracted and chemically synthesized in 1895 . The bronchodilator properties were only later recognized and clinical application for the treatment of asthma began in 1922 (3). Theophylline is prescribed in approximately $35 \%$ of patients with COPD worldwide, and is retained in the Global Initiative for Chronic Obstructive Lung Disease (GOLD) guidelines as a controversial therapy with conflicting data with a number of subsequent trials demonstrating some effect $(2,4-6)$. The role of theophylline in the treatment of asthma and COPD has changed dramatically over the last few decades due in part to the introduction of beta- 2 agonists and inhaled corticosteroids, which demonstrated superior bronchodilatation and anti-inflammatory properties respectively $(3,7)$. The place for theophylline in local clinical practice guidelines has waxed and waned due to its narrow therapeutic index, inherent toxicity and a myriad of drug interactions; despite this, the use of theophylline has persisted largely driven by cost and availability compared to alternate therapies.

\section{Mechanism of action}

The pharmacodynamic action of theophylline remains speculative. A number of potential molecular mechanisms have been suggested which includes phosphodiesterase (PDE) inhibition, adenosine receptor antagonism, upregulation of IL-10, attenuation of IL-8, effects on cell survival, transcription, histone deacetylase activation and enhanced protein phosphatase 2A activity (3) (see Figure 1).

\section{Bronchodilation \& PDE inhibition}

Theophylline has demonstrated large and small human airway bronchodilation in vitro (3). However, the bronchodilator effect is poor at therapeutic concentrations. Intravenous aminophylline has demonstrated efficacy in acute asthma, with a dilatory effect on airway smooth muscle and a prophylactic effect on exercise-induced bronchospasm (8). Theophylline may prove beneficial in COPD patients by reducing air trapping $(3,8,9)$.

Theophylline non-selectively inhibits PDE isoenzymes, which are responsible for degradation of cyclic nucleotides. The net effect of this process is elevated intracellular concentrations of cyclic adenosine monophosphate (cAMP) and cyclic guanosine monophosphate (cGMP) with subsequent smooth muscle relaxation and bronchodilatation (3). Theophylline mediated airway smooth muscle relaxation has been attributed to inhibition of PDE3 activity, an effect only achieved at supratherapeutic concentrations (10). Theophylline also exerts a modest anti-inflammatory effect by preventing the release of cytokines from alveolar macrophages via inhibition of PDE4 activity (10).

\section{Adenosine receptor antagonism}

A further basis for theophylline mediated bronchodilation may be attributed to its antagonistic activity on adenosine receptors, in particular, the $\mathrm{A} 1$ and $\mathrm{A} 2$ subtypes $(3,11)$. Studies have demonstrated that inhaled adenosine constricts bronchial airways in asthmatics by mediating the release of histamine and leukotrienes, an effect that is inhibited by therapeutic concentrations of theophylline (11).

\section{Anti-inflammatory effects}

Theophylline exerts an anti-inflammatory effect in both asthma and COPD, which are achieved at plasma concentrations lower than that required for bronchodilation (3). Low-dose theophylline has been reported to attenuate the hyper-eosinophillic reaction in response to inhaled antigens in mild asthma (12), seen in bronchial biopsies and sputum samples (13). In the context of COPD, theophylline has been associated with a quantitative reduction of neutrophils in sputum supporting an anti-inflammatory effect $(4,14,15)$. A number of mechanisms have been proposed to contribute to these anti-inflammatory effects: 


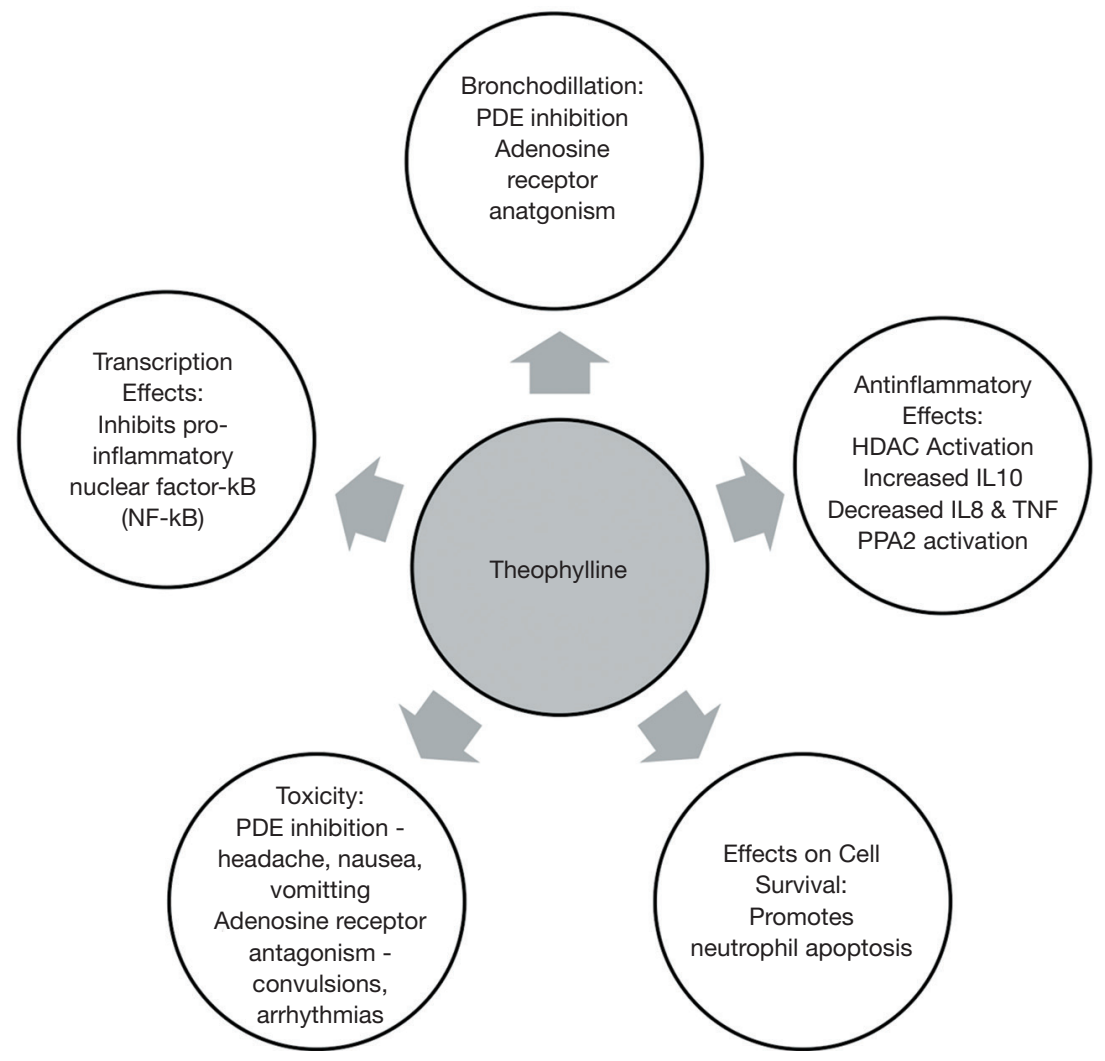

Figure 1 Theophylline: potential mechanisms of action and toxicity.

\section{(I) Increased interleukin-10 (IL-10)}

Asthma and COPD patients have diminished secretion of IL-10, an anti-inflammatory cytokine. Theophylline increases IL-10 release via PDE inhibition (16), albeit at relatively high concentrations, in excess of the low doses effective in asthma (17).

\section{(II) Decreases interleukin-8 (IL-8) and tumor necrosis factor}

IL-8, previously neutrophil chemotactic factor, functions in the recruitment and stimulation of neutrophils and other granulocytes, and in vitro secretion is suppressed by only $10 \mathrm{mM}(1,801 \mu \mathrm{g} / \mathrm{mL})$ of theophylline (18). Notably, this effect is mediated via protein phosphatase $2 \mathrm{~A}(\mathrm{PP} 2 \mathrm{~A})$, and is independent of PDE inhibition and cAMP-dependent MKP-1 up-regulation $(5,6)$.

\section{(III) Protein phosphatase $2 A$ (PP2A)}

$\mathrm{PP} 2 \mathrm{~A}$ is a serine/threonine phosphatase that dephosphorylates several kinases that propagate inflammation, and thus has anti-inflammatory effects $(9,18)$. A study by Patel and Rahman [2016] linked theophylline with PP2A activation in vitro $(16,19)$. PP2A is pivotal in the control of respiratory inflammation, infectious exacerbations, and glucocorticosteroid sensitivity-all conditions that may be improved by theophylline (9).

\section{(IV) Histone deacetylase activation (HDAC)}

HDACs are enzymes that remove acetyl groups from histones, resulting in a tightly coiled conformation (5) and inhibiting the transcription of inflammatory mediators. Low plasma concentrations of theophylline $(<5 \mathrm{mg} /$ L) activates HDACs in states of high oxidative stress $(9,20)$. Oxidative stress in chronic lung diseases (e.g., COPD) leads to activation of inflammatory genes, mucus secretion, and inactivation of protective anti-proteases. This, in conjunction with an altered histone acetylation/ deacetylation balance, leads to increased pro-inflammatory mediators in the lung. Which, in turn, results in the neutralizing of the effectiveness of glucocorticosteroids, as corticosteroids are in part dependent on HDAC2 to turn off pro-inflammatory genes $(21,22)$. Therapeutic and lower concentrations of theophylline activate HDAC and restore its function, thus potentially overcoming this insensitivity to steroids, and reducing inflammation $(5,23)$.

\section{(V) Effects on transcription}

Theophylline, at high plasma concentrations, attenuates 
the activity of inflammatory genes in asthma and COPD patients (24). This is achieved mechanistically by inhibiting the translocation of the pro-inflammatory transcription factor nuclear factor-B (NF-B) (24) into the nucleus by preventing the degradation of the inhibitor, I-Ba (24).

\section{(VI) Effects on cell survival}

Prolonged survival of granulocytes appears to propagate chronic inflammation in COPD. Theophylline promotes apoptosis in neutrophils in vitro through a reduction in the anti-apoptotic protein Bcl-2 (25). Theophylline also causes apoptosis of $\mathrm{T}$ lymphocytes and decreases their survival, again mediated through PDE inhibition (26).

\section{Use of theophylline in clinical disease}

We were unable to find specific data on the use of theophylline in PTBLD when searching published literature or conference abstracts. Therefore, we review the evidence for theophylline in the parallel respiratory diseases of COPD, bronchiectasis and pulmonary hypertension.

\section{Theophylline in COPD}

Theophylline is considered an essential medicine to treat COPD in the Standard Treatment Guidelines and Essential Medicines List for South Africa.

Theophylline has been shown to increase exercise tolerance in patients with COPD by improving respiratory muscle performance (27), increased diffusing capacity and a reduction in peak ventilation (8). In doses that achieve a plasma concentration of $10-20 \mathrm{mg} / \mathrm{L}$, theophylline exhibits a bronchodilatory effect in COPD patients, which is further augmented by the addition of a long acting beta 2 agonist (LABA) (28).

A 2005 meta-analysis of 20 placebo-controlled studies in patients with COPD, which represents the majority of current published clinical efficacy studies, reported that theophylline statistically improved $\mathrm{FEV}_{1}, \mathrm{FVC}, \mathrm{PaO}_{2}$, and $\mathrm{PaCO}_{2}$ levels, and $\mathrm{VO}_{2}$ max (29), while inhibiting cough and improving diaphragmatic function (30,31). Theophylline has also been shown to help improve mucociliary clearance (32).

Reports suggest that monotherapy with low-dose theophylline may reduce COPD exacerbations by approximately $50 \%$ over 1 year (6). In vitro and $e x$ vivo studies suggest that COPD associated inflammation is partially refractory to glucocorticosteroid therapy. It has been proposed that lower plasma concentrations of theophylline $(1-5 \mathrm{mg} / \mathrm{L})$ may augment the anti-inflammatory effects of corticosteroids in COPD by enhancing or restoring HDAC activity in airway macrophages, resulting in suppression of inflammatory genes and enhancement of the anti-inflammatory effects of glucocorticoids $(5,29)$. Although there are laboratory data supporting the steroid sparing effects in COPD, current literature from clinical trials is conflicting. Low-dose theophylline has been shown to enhance the recovery from acute exacerbations of COPD, and is associated with reduced inflammation and increased HDAC activity (33). A small study of 30 patients with moderate COPD in a double-dummy, randomised, double-blind, parallel study, demonstrated that low-dose theophylline elicited a greater anti-inflammatory effect and improvement in FEV1 when added to an inhaled corticosteroid than either drug alone (34). A more recent randomised control trial in 1,567 patients, failed to demonstrate a reduced number of COPD exacerbations over a 1 year period, when low dose theophylline was added to inhaled corticosteroid (7). However, a post hoc sub-analysis revealed that there were more COPD related hospital admissions in the placebo group compared to the theophylline-treated group (7). Thus, although there appears evidence for synergism of theophylline with glucocorticosteroids in COPD, current literature remains conflicting in this regard.

\section{Bronchiectasis}

Bronchiectasis is defined as abnormal, permanent dilation of the airways for which there are many causes. Postinfectious causes remain the most frequent aetiology for bronchiectasis in LMICs, of which tuberculosis is the most important locally (35).

Bronchiectasis predisposes to recurrent infections, inflammation and further damage to the airways, in turn perpetuating the cycle of recurrent infections and damage in the airways. Frequently, bronchiectasis demonstrates airflow obstruction, and theophylline with the proposed therapeutic bronchodilatory and anti-inflammatory mechanisms described above, may have some theoretical utility in this disease. Currently, there is no evidence for or against the use of theophylline in bronchiectasis. A Cochrane review conducted in 2000, investigating the utility of oral methylxanthines in the treatment of bronchiectasis, failed to identify any trials supporting the use of theophylline in bronchiectasis (36). A clinical trial conducted by Zhong et al., 2015, which evaluated the clinical efficacy and safety of theophylline in the treatment 
Table 1 Drug interactions and factors affecting theophylline clearance (3)

\begin{tabular}{ll}
\hline Increased clearance & Decreased clearance \\
\hline Enzyme inducers (rifampicin, phenobarbitone, ethanol) & Enzyme inhibition (cimetidine, erythromycin, ciprofloxacin, allopurinol, zileuton) \\
Smoking (tobacco, cannabis) & Congestive heart failure \\
High protein, low carbohydrate diet & Liver disease \\
Barbecued meat & Pneumonia \\
Childhood & Viral Infection \\
& Vaccination (immunization) \\
& High carbohydrate diet \\
& Old age \\
\hline
\end{tabular}

Reproduced with permission: Prof PJ Barnes (Margaret Turner-Warwick Professor of Medicine; Airway Disease Section, National Heart \& Lung Institute, Dovehouse St, London SW3 6LY, UK).

of non-cystic fibrosis bronchiectasis, reached completion in study objective, however, the results of the study have yet to be published (37). Thus, we currently have no evidence to either support or refute the use of theophylline in the management of bronchiectasis.

\section{Theophylline in pulmonary hypertension}

Tuberculosis has not been cited as a cause of pulmonary hypertension in major guidelines (38), however local clinical experience suggests otherwise and potentially may be the most important cause of group 3 pulmonary hypertension (due to chronic lung disease) locally (39). Theophylline may be beneficial in treating pulmonary hypertension. Early studies suggested that intravenous aminophylline reduces pulmonary artery pressures and pulmonary vascular resistance with associated increases in right and left ventricular ejection fraction in COPD patients (40). Oral long-acting theophylline is thought to produce a similar and chronic improvement in biventricular performance. Postulated mechanisms by which theophylline augments right and left ventricular systolic function includes a reduction in ventricular afterload and positive inotropy (40). Theophylline has not been studied in isolated pulmonary hypertension, but it is possible that theophylline may demonstrate utility in patients with a combination of COPD, pulmonary artery hypertension, and right or left heart failure. However, patients with right ventricular failure may have decreased clearance of theophylline, warranting careful monitoring of plasma levels (see below) (41).

\section{Relevant pharmacology}

\section{“Theophylline in the PTBLD” (Pharmacokinetics)}

Theophylline is considered by many clinicians as a "dirty drug", due perceived toxicity, side-effects, and intra- and interpatient variability. There appears to be a linear relationship between serum theophylline concentrations and improved airway function. Bronchodilator effects are negligible below $10 \mathrm{mg} / \mathrm{L}$, whilst side effects are commonplace above $25 \mathrm{mg} / \mathrm{L}$ and therefore, the therapeutic range for theophylline has been reported as 10 to $20 \mathrm{mg} / \mathrm{L}$ (3). Notably, non-bronchodilator effects of theophylline are achieved at concentrations of less than $10 \mathrm{mg} / \mathrm{L}$ (3). The attainment of therapeutic concentrations is influenced mainly by population and interpatient variances in clearance, which is, in turn, determined primarily by hepatic metabolism. Theophylline is rapidly and completely absorbed and is metabolised in the liver by the cytochrome P450 microsomal enzyme system (CYP1A2 in particular). The hepatic metabolism of theophylline is influenced by several pharmacokinetic factors, concurrent disease states, as well as drug interactions (see Table 1) (3). Increased clearance is seen in children and in cigarette and marijuana smokers (3). For example; concomitant administration of CYP450 enzyme inducers phenytoin, or rifampicin, result in low theophylline plasma levels, thus higher doses may be required. Decreased clearance of theophylline may be seen with liver disease, and heart failure, and therefore appropriate dose reduction and monitoring of plasma levels is required (24). There is a paucity of data on the pharmacogenetics of theophylline. However, a Turkish 
study conducted in COPD patients found that the $\mathrm{T}$ allele of rs35694136 is linked with decreased metabolism of theophylline (42). Furthermore, data from a study conducted in Japanese asthmatic patients (replicated by a study in Korean asthmatic patients), demonstrated that genotypes AA and AG of rs2069514 in the CYP1A2 gene are associated with decreased metabolism of theophylline (43-45). Various single nucleotide polymorphisms (SNPs) in the CYP2E1 and $C Y P 1 A 2$ promoter regions have been linked to altered hepatic metabolism and lower plasma theophylline levels, respectively (45). There are no pharmacogenetic studies on theophylline in sub-Saharan Africa.

\section{Potential pitfalls}

\section{Drug interactions (see Table 1)}

A reduction in theophylline clearance occurs with the co-administration of the following drugs: erythromycin, quinolone antibiotics (excluding ofloxacin), allopurinol, cimetidine, selective serotonin uptake inhibitors (fluvoxamine), and the 5-lipoxygenase inhibitor zileuton, all of which induce the CYP1A2 isoenzyme. With respect to the macrolide class; similar interactions are seen with clarithromycin, but there is no reported interaction with azithromycin (46). Vaccinations (influenza immunizations) have also been reported to reduce clearance (3).

Due to the wide variations in theophylline hepatic metabolism and clearance, careful therapeutic drug monitoring and dose is required. Plasma concentrations should ideally be measured 4 hours after the last dose with slow-release preparations (3).

\section{Side effects and toxicity}

In high-income country settings, toxic exposures to theophylline have decreased significantly since its management for asthma and COPD has declined. From 1985 to 1995 in the United States, theophylline poisoning requiring haemodialysis or haemoperfusion accounted for 49 cases per one million poison centre calls, compared to the 6 cases per one million poison centre calls from 1996 to 2005. According to the American Association of Poison Control Centres, there were 133 exposures to theophylline in 2014 (47). A South African toxicovigilance study conducted by Veale et al., 2013 found that theophylline overdose comprised only $1.5 \%$ of the total medicine related exposures (48).
Theophylline has a narrow therapeutic window with toxic effects occurring when serum theophylline levels surpass the levels in the therapeutic range (usually $>20 \mathrm{mg} / \mathrm{L}$ ). This occurs primarily by intentional overdose or unintentionally when metabolism and/or clearance of theophylline is altered due to certain physiological stressors. The main toxic effects may be summarized as follows $(3,47)$ :

* General: Agitation, irritability, restlessness;

* Cardiovascular: Sinus tachycardia, ventricular tachycardia atrial fibrillation, supraventricular tachycardia, hypotension, cardiac arrest;

* Respiratory: Tachypnoea, acute lung injury, respiratory alkalosis;

* Gastrointestinal: Nausea, vomiting, abdominal pain;

* Neurological: Tremors, hallucinations, seizures.

The inhibition of PDE isoenzymes frequently result in headache, nausea and vomiting, increased acid secretion, and gastroesophageal reflux, whilst diuresis has been attributed to adenosine receptor antagonism. Convulsions and cardiac arrhythmias may occur at high plasma concentrations and are associated with adenosine A1Areceptor antagonism. Side effects may be mitigated by slow dose titration until therapeutic concentrations are achieved. Furthermore, avoiding the toxic effects of theophylline can achieved by appropriate therapeutic drug monitoring, patient education and prescriber pharmacovigilance. The clinical presentation and management of non-accidental overdose of theophylline is beyond the scope of this review. Given the above side effect profile of theophylline and the potential efficacy of low doses in context of PTBLD, theophylline may prove to be a safe therapeutic and costeffective agent for the management of this disease entity. Currently no direct comparator or cost-effectiveness studies exist that compare theophylline with other medications used in pulmonary hypertension. However, if found of benefit, the cost saving of theophylline (USD 21.00 for one month at $200 \mathrm{mg}$ ) compared to, for example, oral sildenafil (USD 282.00 for one month at $50 \mathrm{mg}$ ) could be an important consideration in places where PTBLD is prevalent.

The use of lower doses than that used in chronic obstructive lung disease or asthma limits the need for intensive therapeutic drug monitoring and most of the drug side effects which are dose related. We do however advocate therapeutic drug monitoring for some patients with PTBLD who may have altered drug metabolism (liver disease, renal disease) and may have drug-drug interactions secondary to use of concomitant enzyme inhibiting/inducing drugs. 
We advocate comprehensive patient education with respect to side effect awareness, informing prescribers of changes in drug regimens and the use of concomitant over-thecounter medications. A national drug registry may help with monitoring of adverse effects and efficacy of low dose theophylline in TB associated lung disease.

\section{Conclusions}

Theophylline for chronic respiratory diseases remains controversial with more effective agents promoted for COPD, while no data exists in bronchiectasis or pulmonary hypertension, yet it remains both affordable, available and prescribed in many low- and middle-income countries, including South Africa. The narrow therapeutic index, side-effect profile and drug-drug interactions, need to be balanced against anti-inflammatory effects at lower-doses and safety facilitated by therapeutic drug monitoring. The anti-inflammatory, bronchodilatory and possible pulmonary-vascular effects make it an attractive potential agent for PTBLD, which has overlapping phenotypes of bronchiectasis, chronic airflow obstruction and pulmonary hypertension. Given the paucity of evidence supporting the use of theophylline in patients with PTBLD, robust clinical trials are needed.

\section{Acknowledgments}

Funding: None.

\section{Footnote}

Conflicts of Interest: All authors have completed the ICMJE uniform disclosure form (available at http://dx.doi. org/10.21037/jtd-20-1298). The authors have no conflicts of interest to declare.

Ethical Statement: The authors are accountable for all aspects of the work in ensuring that questions related to the accuracy or integrity of any part of the work are appropriately investigated and resolved.

Open Access Statement: This is an Open Access article distributed in accordance with the Creative Commons Attribution-NonCommercial-NoDerivs 4.0 International License (CC BY-NC-ND 4.0), which permits the noncommercial replication and distribution of the article with the strict proviso that no changes or edits are made and the original work is properly cited (including links to both the formal publication through the relevant DOI and the license). See: https://creativecommons.org/licenses/by-nc-nd/4.0/.

\section{References}

1. Ravimohan S, Kornfeld H, Weissman D, et al. Tuberculosis and lung damage: from epidemiology to pathophysiology. Eur Respir Rev 2018;27:170077.

2. GOLD. Gold 2019. Glob Inititiative Chronic Obstr Lung Dis. 2019.

3. Barnes PJ. Theophylline. Am J Respir Crit Care Med 2013;188:901-6.

4. Kobayashi M, Nasuhara Y, Betsuyaku T, et al. Effect of low-dose theophylline on airway inflammation in COPD. Respirology 2004;9:249-54.

5. Cosio BG, Tsaprouni L, Ito K, et al. Theophylline restores histone deacetylase activity and steroid responses in COPD macrophages. J Exp Med 2004;200:689-95.

6. Zhou Y, Wang X, Zeng X, et al. Positive benefits of theophylline in a randomized, double-blind, parallelgroup, placebo-controlled study of low-dose, slowrelease theophylline in the treatment of COPD for 1 year. Respirology 2006;11:603-10.

7. Devereux G, Cotton S, Fielding S, et al. Effect of Theophylline as Adjunct to Inhaled Corticosteroids on Exacerbations in Patients With COPD: A Randomized Clinical Trial. JAMA 2018;320:1548-59.

8. Voduc N, Alvarez GG, Amjadi K, et al. Effect of theophylline on exercise capacity in COPD patients treated with combination long-acting bronchodilator therapy: a pilot study. Int J Chron Obstruct Pulmon Dis 2012;7:245-52.

9. Patel BS, Rahman MM, Rumzhum NN, et al. Theophylline Represses IL-8 Secretion from Airway Smooth Muscle Cells Independently of Phosphodiesterase Inhibition. Novel Role as a Protein Phosphatase 2A Activator. Am J Respir Cell Mol Biol 2016;54:792-801.

10. Rabe KF, Magnussen H, Dent G. Theophylline and selective PDE inhibitors as bronchodilators and smooth muscle relaxants. Eur Respir J 1995;8:637-42.

11. Polosa R, Blackburn MR. Adenosine receptors as targets for therapeutic intervention in asthma and chronic obstructive pulmonary disease. Trends Pharmacol Sci 2009;30:528-35.

12. Sullivan P, Bekir S, Jaffar Z, et al. Anti-inflammatory effects of low-dose oral theophylline in atopic asthma. Lancet 1994;343:1006-8. 
13. Lim S, Tomita K, Caramori G, et al. Low-dose theophylline reduces eosinophilic inflammation but not exhaled nitric oxide in mild asthma. Am J Respir Crit Care Med 2001;164:273-6.

14. Culpitt SV, de Matos C, Russell RE, et al. Effect of theophylline on induced sputum inflammatory indices and neutrophil chemotaxis in chronic obstructive pulmonary disease. Am J Respir Crit Care Med 2002;165:1371-6.

15. Kanehara M, Yokoyama A, Tomoda Y, et al. Antiinflammatory effects and clinical efficacy of theophylline and tulobuterol in mild-to-moderate chronic obstructive pulmonary disease. Pulm Pharmacol Ther 2008;21:874-8.

16. Mascali JJ, Cvietusa P, Negri J, et al. Anti-inflammatory effects of theophylline: modulation of cytokine production. Ann Allergy Asthma Immunol 1996;77:34-8.

17. Oliver B, Tomita K, Keller A, et al. Low-dose theophylline does not exert its anti-inflammatory effects in mild asthma through upregulation of interleukin-10 in alveolar macrophages. Allergy 2001;56:1087-90.

18. Iiboshi H, Ashitani J, Katoh S, et al. Long-term treatment with theophylline reduces neutrophils, interleukin- 8 and tumor necrosis factor-alpha in the sputum of patients with chronic obstructive pulmonary disease. Pulm Pharmacol Ther 2007;20:46-51.

19. Hirano T, Yamagata T, Gohda M, et al. Inhibition of reactive nitrogen species production in COPD airways: comparison of inhaled corticosteroid and oral theophylline. Thorax 2006;61:761-6.

20. Ito K, Lim S, Caramori G, et al. A molecular mechanism of action of theophylline: Induction of histone deacetylase activity to decrease inflammatory gene expression. Proc Natl Acad Sci U S A 2002;99:8921-6.

21. Zuccaro L, Cox A, Pray C, et al. Histone deacetylase activity and recurrent bacterial bronchitis in severe eosinophilic asthma. Allergy 2016;71:571-5.

22. Dombrowsky H, Uhlig S. Steroids and histone deacetylase in ventilation-induced gene transcription. Eur Respir J 2007;30:865-77.

23. Ranjani R, Vinotha ATS. A prospective randomized controlled study: Theophylline on oxidative stress and steroid sensitivity in chronic obstructive pulmonary disease patients. Int J Pharm Investig 2017;7:119-24.

24. Zhang ZY, Kaminsky LS. Characterization of human cytochromes P450 involved in theophylline 8-hydroxylation. Biochem Pharmacol 1995;50:205-11.

25. Chung IY, Nam-Kung EK, Lee NM, et al. The downregulation of Bcl-2 expression is necessary for theophylline-induced apoptosis of eosinophil. Cell
Immunol 2000;203:95-102.

26. Ohta K, Yamashita N. Apoptosis of eosinophils and lymphocytes in allergic inflammation. J Allergy Clin Immunol 1999;104:14-21.

27. Murciano D, Auclair MH, Pariente R, et al. A randomized, controlled trial of theophylline in patients with severe chronic obstructive pulmonary disease. N Engl J Med 1989;320:1521-5.

28. ZuWallack RL, Mahler DA, Reilly D, et al. Salmeterol plus theophylline combination therapy in the treatment of COPD. Chest 2001;119:1661-70.

29. Cosío BG, Shafiek H, Iglesias A, et al. Oral Low-dose Theophylline on Top of Inhaled Fluticasone-Salmeterol Does Not Reduce Exacerbations in Patients With Severe COPD: A Pilot Clinical Trial. Chest 2016;150:123-30.

30. Aubier M. Effect of theophylline on diaphragmatic muscle function. Chest 1987;92:27S-31S.

31. Ram FS, Jardin JR, Atallah A, et al. Efficacy of theophylline in people with stable chronic obstructive pulmonary disease: a systematic review and meta-analysis. Respir Med 2005;99:135-44.

32. Sutton PP, Pavia D, Bateman JR, et al. The effect of oral aminophylline on lung mucociliary clearance in man. Chest 1981;80:889-92.

33. Cosio BG, Iglesias A, Rios A, et al. Low-dose theophylline enhances the anti-inflammatory effects of steroids during exacerbations of COPD. Thorax 2009;64:424-9.

34. Ford PA, Durham AL, Russell RE, et al. Treatment effects of low-dose theophylline combined with an inhaled corticosteroid in COPD. Chest 2010;137:1338-44.

35. O'Donnell AE. Medical management of bronchiectasis. J Thorac Dis 2018;10:S3428-35.

36. Steele K, Greenstone M, Lasserson JA. Oral methylxanthines for bronchiectasis. Cochrane Database Syst Rev 2001;(1):CD002734.

37. NCT01684683. The Effect of Theophylline in the Treatment of Bronchiectasis. Available online: Https:// clinicaltrials.gov/show/nct01684683

38. Simonneau G, Montani D, Celermajer DS, et al. Haemodynamic definitions and updated clinical classification of pulmonary hypertension. Eur Respir J 2019;53:1801913.

39. Allwood BW, Maarman GJ, Kyriakakis CG, et al. Postpulmonary tuberculosis complications in South Africa and a potential link with pulmonary hypertension: Premise for clinical and scientific investigations. S Afr Med J 2018;108:12339.

40. Matthay RA. Effects of theophylline on cardiovascular 
performance in chronic obstructive pulmonary disease. Chest 1985;88:112S-7S.

41. Barbera J, Peinado VI, Santos S. Pulmonary hypertension in chronic obstructive pulmonary disease. Eur Respir J 2003;21:892-905.

42. Uslu A, Ogus C, Ozdemir T, et al. The effect of CYP1A2 gene polymorphisms on Theophylline metabolism and chronic obstructive pulmonary disease in Turkish patients. BMB Rep 2010;43:530-4.

43. Obase Y, Shimoda T, Kawano T, et al. Polymorphisms in the CYP1A2 gene and theophylline metabolism in patients with asthma. Clin Pharmacol Ther 2003;73:468-74.

44. Yoon Y, Park HD, Park KU, et al. Associations between CYP2E1 promoter polymorphisms and plasma 1,3-dimethyluric acid/theophylline ratios. Eur J Clin

Cite this article as: Karamchand S, Wiiliams M, Naidoo P, Decloedt E, Allwood B. Post-tuberculous lung disease: should we be using Theophylline? J Thorac Dis 2021;13(2):1230-1238. doi: $10.21037 /$ jtd-20-1298
Pharmacol 2006;62:627-31.

45. Whirl-Carrillo M, McDonagh EM, Hebert JM, et al. Pharmacogenomics knowledge for personalized medicine. Clin Pharmacol Ther 2012;92:414-7.

46. Nahata M. Drug interactions with azithromycin and the macrolides: an overview. J Antimicrob Chemother 1996;37 Suppl C:133-42.

47. Journey J, Bentley T. Theophylline Toxicity. Treasure Island (FL): StatPearls, 2019.

48. Veale DJ, Wium CA, Müller GJ. Toxicovigilance. II: A survey of the spectrum of acute poisoning and current practices in the initial management of poisoning cases admitted to South African hospitals. S Afr Med J 2013;103:298-303. 